BMJ Open

Diabetes

Research

\& Care

\title{
Experiences and challenges of adults living with type 2 diabetes mellitus presenting at the University Teaching Hospital in Lusaka, Zambia
}

\author{
Kunda Faith Mwila (D , , ${ }^{1,2}$ Phoebe Albina Bwembya, ${ }^{1}$ Choolwe Jacobs ${ }^{3}$
}

\section{To cite: Mwila KF,}

Bwembya PA, Jacobs C. Experiences and challenges of adults living with type 2 diabetes mellitus presenting at the University Teaching Hospital in Lusaka, Zambia. BMJ Open Diab Res Care 2019;7:e000497. doi:10.1136/ bmjdrc-2017-000497

Received 14 January 2019 Revised 23 September 2019 Accepted 25 October 2019
Check for updates

C) Author(s) (or their employer(s)) 2019. Re-use permitted under CC BY-NC. No commercial re-use. See rights and permissions. Published by BMJ.

${ }^{1}$ Department of Population studies and Nutrition, University of Zambia School of Medicine (Public Health), Lusaka, Zambia ${ }^{2}$ Department of Pediatrics and Child Health, The University Teaching Hospital, Lusaka, Zambia

${ }^{3}$ Department of Epidemiology and Bio-Statics, University of Zambia School of Medicine, Lusaka, Lusaka, Zambia

Correspondence to Kunda Faith Mwila; everlynmwila@gmail.com

\section{ABSTRACT}

Objective The study explored the experiences and challenges of adults living with type 2 diabetes mellitus (T2DM) presenting at the University Teaching Hospital (UTH) in Lusaka.

Research design A qualitative descriptive study was conducted. The research relied on purposive sampling to select 28 participants for in-depth interviews. Participants were interviewed during clinical visits at UTH.

Results Views from participants showed that some adults living with T2DM experienced physical and mental illnesses. Participants' views reflected that their livelihood with T2DM was influenced by family support, poor or non-adherence to treatment guidelines and access to information, education and communication materials. The most important challenges reported were psychosocial and financial.

Conclusion The study concluded that some adults living with T2DM experience a lot of physical sicknesses and their challenges, especially of the psychosocial nature may require professional attention. However, particular attention should be paid to the patient's self-care and psychosocial therapy. Self-care protocols should be tailored to complement the different types of patients with diabetes and improve their quality of life.Cite Now

\section{INTRODUCTION}

Globally, an estimated 422 million adults are living with diabetes, according to the 2016 data from the WHO; compared with the 108 million in $1980 .{ }^{1}$ Type 2 diabetes mellitus (T2DM) is the most prevalent and steadily increasing chronic metabolic disorder. An estimated $80 \%$ of people with T2DM are living in low-income and middle-income countries. ${ }^{2}$

T2DM is the most common form of diabetes $(90 \%-95 \%)$ in sub-Saharan Africa. ${ }^{3}$ In Zambia, T2DM is becoming an emerging public health concern. As of 2008, estimates indicate that Zambia may be one of the countries with a high prevalence of noncommunicable diseases in the world. ${ }^{4} \mathrm{~A}$ population-based survey conducted in Zambia in 2012 found the prevalence of T2DM to be $2.1 \%$ among men and $3.0 \%$ among women. ${ }^{5}$

\section{Significance of this study}

What is already known about this subject?

- Type 2 diabetes mellitus (T2DM) is one of the leading causes of mortality worldwide. Diabetic treatment guidelines are difficult to follow and people living with T2DM find it difficult to manage the condition.

What are the new findings?

- Some adults living with T2DM in Zambia experience physical sicknesses, mental ailments, poor family support and adherence and inadequate information, education and communication. People living with T2DM are challenged financially and psychosocially and in the use of daily services and products such as public transportation and nutrition (dietary needs). Someone's work schedule also presents further challenges in people living with T2DM.

How might these results change the focus of research or clinical practice?

- This study has served as a starting point for further diabetes mellitus research in the Zambia and intends to inform practice and policy on the experiences and challenges tackled by people living with T2DM which, in turn, may improve the management of diabetes mellitus among adults.

T2DM is a chronic disease and patients may have difficulties, mental problems or stress as a result of efforts to comply with treatment guidelines. ${ }^{6}$ Studies have shown that depression was a common comorbid health problem among Zambian people with T2DM who face significant challenges in accessing diagnosis and treatments contributing to high mortality and prevalence of complications in sub-Saharan Africa. ${ }^{78}$ Studies in Zambia on T2DM are scarce leaving a gap that needs to be filled. ${ }^{9}$ In Zambia, efforts are being made to prevent complications and help patients cope with the condition. However, the experiences and challenges of adults with T2DM are not known. 


\section{RESEARCH DESIGN AND METHODS}

\section{Design}

A qualitative descriptive study was used to conduct this study between 2015 and 2017 . The study was conducted to explore experiences and challenges of adults living with T2DM presenting at the University Teaching Hospital (UTH) in Lusaka.

\section{Site and participants}

The study was conducted at UTH, the largest tertiary referral and teaching hospital in Zambia. The hospital admits the largest number of patients living with diabetes mellitus. Adult patients with T2DM attending the outpatient and inpatient clinic in the department of Internal Medicine at UTH made up the study population. The study included male and female adults aged from 20 to 75 years with known T2DM. The study excluded patients who had severe medical illness (physical or mental handicaps, bedridden or pregnant) and patients that did not give consent to take part in the research.

\section{Sampling procedure}

Participants (adults with diabetes) were recruited using a purposive sampling method. This was carried out with the help of hospital clerks that identified the people living with T2DM using their hospital files. All people with T2DM were informed of the study while waiting for consultation at their clinical appointment at clinic 5 by the hospital clerks. To realize the objectives of the study, information was also obtained from hospital patient records. The total sample size was 28 .

\section{Data collection, management and analysis}

In-depth interviews were conducted with the aid of a structured topic guide and digital audio recorder. ${ }^{10}$ The topic guide/questions were developed using the aims and objectives of the study in combination with qualitative research literature of guidelines on how to conduct oneto-one interviews. ${ }^{1112}$ Data collection tools were pretested at the UTH in clinic 5. Interviews were conducted in English, Bemba and Nyanja. Bemba and Nyanja are local languages widely spoken in Lusaka District. Data collection was concluded based on thematic saturation.

All recorded interviews were transcribed into electronic format by listening to the recordings thoroughly and writing down everything. ${ }^{13}$ The transcripts and other relevant documents were then translated into English. To ensure accurate translation of information, one of the co-authors (MK) re-read both local language and English transcripts to cross-check the interpretations for any errors. The edited transcripts were organized as primary documents in Atlas.ti7. ${ }^{14}$ Using the code and memo managers in Atlas.ti7, ${ }^{15}$ codes were created and defined. Then quotations were added to these codes and output was produced. This output was printed out and the codes categorized concluding the first cycle of coding.

The categorized codes were compared with one another to recognize similarities and the information was used to generate and summarize major and sub-themes from the given responses ending the second level of coding. ${ }^{16} 17$ Then, the third level of coding to further analyze the results from the first two stages and discover how the new themes and sub-themes interrelate with one another was also conducted. ${ }^{1617}$ Two major themes emerged from the analysis namely experiences and challenges. The data transcription, coding and analysis were conducted by two individuals, the primary author (MK) and a co-author (CJ) who discussed any discrepancies until consensus was reached. The authors documented the potential findings and implications of the study in analytic memos/ notebooks which were useful for studying the emergent patterns, themes and concepts.

\section{Ethical considerations}

Formal permission was obtained from the UTH management to conduct the research in the hospital premises. The participants were approached by hospital staff when they came to the hospital for review/consultations with doctors. Potential respondents were requested to visit the research office just before departure. An information sheet was provided to participants that explained all the necessary information concerning procedures and objectives of the study. Written informed consent together with permission to carry out voice recordings during interviews was sought from participants before the interviews commenced.

The respondents were assured of privacy and confidentiality of data collected. No identifying information linked to them, nor the information they submitted was shared publicly except for the anonymized information that appears in the results section. The interview audio and written transcripts and all documents related to this project are encrypted and stored on a password protected private computer.

\section{RESULTS}

Out of the 28 respondents aged between 38 years and 75 years, 11 were men and 17 were women. Two of the females were disabled (blind and unable to walk). Of the 28 participants, 17 were in informal employment and nine were retired. In all, 21 of the respondents were married and six were never married. Furthermore, education levels of the respondents in the research where that 15 of them went to secondary school and seven had reached primary school, while four attained tertiary education and only two had no schooling of any kind. The shortest time period lived with diabetes was 2 months and the longest was 22 years.

The two major themes experiences and challenges are discussed below.

\section{Theme 1: experiences}

\section{Physical experiences}

Although some respondents said that they experienced healthy lives with diabetes, most of the respondents reported that they were weak and fell ill often. Their 
physical well-being/immune system is weakened causing their own bodies to be vulnerable to infections. Some medical complaints that the respondents mentioned ranged from conditions that affect the chest cavity such as difficulty with breathing in the night, lower limbs such as numbness on hands and legs and mostly fatigue, sight, sexual and other parts of the body.

My life hasn't been healthy since my diabetes mellitus diagnosis. I am sick mostly. I wake upjust feeling powerless sometimes. I was able to do a lot of work when I woke up but now I can't even sell my products because I have no strength. (Participant 7)

Other illnesses included headaches and heat episodes. Most of the respondents reported that they felt fatigued and could not work as they used to before the diabetes diagnosis. However, a few respondents reported that they experience sharp pains, abdominal pains and bloated stomach.

In the lower parts of the body that consists of the legs, some respondents complained of painful legs and hands and also swelling legs.

\section{Sight and sexual dysfunction}

Some respondents reported having vision problems like poor sight and total blindness, whereas other respondents reported that they were experiencing sexual problems such as loss of libido particularly for men and vaginal itchiness and discharge for women.

My sight is not very good. I read with glasses. (Participant 4).

It is difficult to do more than one round of sexual intercourse. (Participant 5).

\section{Blood sugar levels}

Some people living with T2DM experience mostly elevated blood sugar levels because of their surrounding and how their body mechanisms function. These sugar levels lead to doctors' visits and hospital admissions. Narrations included how most of the respondents experienced sicknesses and hyperglycemia despite their good or poor adherence to treatment guidelines. Other respondents found their clinical follow-ups inconveniencing though helpful.

Mostly I experience high blood sugar levels. (Participant $3)$.

\section{Inadequate information education and communication}

The respondents reported that they had inadequate information, education and communication messages about T2DM. This is evident as some respondents narrated that they needed more information on the condition.

When will I get cured from this condition? (Participant 1) Where can we get information about what causes diabetes and to cure it? (Participant 28)
This shows that the patients do not have enough information concerning their condition. Information, education and communication materials provide vital information on any topic. In diabetes, for example, these materials may define the condition/disease, prevention, symptoms, causes, treatment, complications and any other relevant information about T2DM. In some cases, T2DM has been mistaken for things like witchcraft. A male respondent narrated how he is living with T2DM and how his father died exhibiting the same symptoms years ago but was not diagnosed with T2DM. Instead, the whole family thought that he had been bewitched. These wrong conclusions would not have been made if patients had enough information on their condition.

\section{Poor/non-adherence to diabetic treatment guidelines}

The participants were asked to rate their compliance with treatment guidelines using a scale from 0 to 10 where 0 indicated non-compliance and 10 was the highest level of compliance. The results indicate that the respondents tried by all means to comply with treatment guidelines such that the lowest level of compliance indicated was $50 \%$ and the highest was $100 \%$.

The findings showed that the respondent's surrounding environment (physical and social) presented circumstances that made it difficult to take medication on time, exercise regularly and eat the right food. The most challenging experience reported is that it is difficult for respondents that are employed to take medicine and eat regularly because of the nature of their employment that keeps them away from restaurants with healthy food. For example, a respondent reported that sometimes she feels lazy to take the medicine because she takes it every day. Another respondent compared adherence to treatment guidelines of diabetes as being in a prison where they are forced to take medicine every day and are restricted to what food they eat even though they want to eat something else that is being craved for.

Sometimes I eat in good time. It just depends on circumstances. I do manage to inject myself on time but sometimes I feel lazy and just say, 'I will sleep like this without injecting tonight.' And I take the injection in the morning. (Participant 9).

\section{Family support}

This was measured using a scale of 0 to 10 where 0 meant the respondent was never supported by any family members and 10 meant the highest/greatest level of family support received. The results showed that 22 of the respondents are emotionally supported by family and friends in their living with the condition while six do not receive the support. The respondents that do not get family support experience a life of anguish as they are emotionally challenged by their loved ones.

I have it very tough as a diabetic. My children really give a hard time, they tease me. Sometimes they get my clinical card and play with it." (Respondent starts to cry). When 
I buy these DM recommended foods, they complain that I am buying expensive food. When in actual sense the same food doesn't even taste nice. So my heart is really troubled. (Participant 20).

\section{Mental distress}

The results also point to the fact that some people living with T2DM undergo diabetes-related distress. The respondents reported having experienced emotional/ mental challenges and situations that presented feelings of frustration, anger, stress and depression. Even though some respondents described not having vital emotions, most of them have gone through a number of psychosocial challenges but have learnt to prevent or cope with them with time.

I get very angry. I even get depressed sometimes". (Participant breaks out crying) "I hate myself. I feel very bad because I am the only one that is sick. Everyone else is healthy. (Respondent continues to sob). (Participant 20).

\section{Theme 2: challenges}

\section{Psychosocial}

People living with T2DM may undergo diabetes-related stress and psychosocial challenges in the way they relate with other people. A few respondents reported having no psychosocial challenges while seventeen of them expressed feelings of frustration, stress, depression and anger toward or brought about by their social environment. For example, a number of respondents narrated being angry at workmates and family members fueled by diabetes-related stress. Thoughts of how to get medicine when it runs out or how to purchase the required food also increased stress levels and encouraged depression.

Psychosocial challenges were measured using a scale of 0 to 10 where 0 meant the respondent did not experience any emotional changes that could be attributed to T2DM and 10 meant the highest/greatest level of experience of emotional changes that could be attributed to T2DM. In all, 21 of the participants indicated levels of psychological challenges that ranged from five to eight. This clearly shows some concerning levels of psychological challenges.

Further psychosocial challenges were explained by respondents who said that taking medicine every day and attending parties without eating the food served there aroused feelings of depression and seclusion from social circles. However, the respondents that have lived longer with diabetes said that they have resorted to ignoring all psychological emotions to maintain optimum health.

I get depressed sometimes because if one goes to a function, one can't eat the food they serve their freely. So it makes me feel out of place. Also, I am forced to eat that food despite the food not being healthy for me. (Participant 15).

\section{Financial challenges}

The findings also showed that living with diabetes mellitus may present financial challenges. Even though some respondents explained how they struggle to buy medicine and food recommended as part of their nutritional therapy, others still manage to purchase the required food and medicine. Despite UTH providing medicine, it is not enough to last until the next review date. This forced patients to purchase medication to supplement what was received from the hospital.

They give medicine here but it doesn't last long, so I buy to supplement the shortage. The medication is a bit expensive. But I have no option but to buy. (Participant 12).

The findings also showed that diabetic nutritional therapy is expensive as reported by respondents. The respondents also reported that some diabetic foods are scarce and the nutrients content do not work the way they are supposed to. That is to say some foods do not reduce the blood sugar levels in the patients as the packaging labels indicate.

It's difficult to get the diabetes recommended food because I don't have enough money. I sell tomatoes by the roadside. (Participant 14).

\section{Transportation}

Another challenge faced by some people living with T2DM involves traveling by public transportation. A male respondent that expressed frustration narrated how bad it felt to travel on a public bus over a long distance with the rest of the passengers that are only interested in reaching their destination.

I manage to eat in time. When travelling, like when I was on my way coming here on the bus, I couldn't take any liquids because sometimes I have to tell the driver to stop the bus. (Participant 19)

\section{DISCUSSION}

This study explored experiences and challenges of adults living with T2DM in Lusaka. Based on the findings, the experiences of adults living with T2DM were categorized as physical, mental, poor/non-adherence, family support and inadequate information, education and communication. The physical experiences were those that were associated with the chest cavity and lower limbs, sight, sexual dysfunction, high blood sugar and other general illnesses. These experiences were influenced by the environment surrounding some adults living with T2DM among respondents. This study also revealed financial and transportation challenges experienced by some people living with T2DM.

Fatigue is a common and distressing complaint among people with T2DM as shown by responses that were given in this study. In a review of the literature about diabetesrelated fatigue, the results indicated that diabetes presents many potential pathways for fatigue, but focused 
studies on this symptom are rare. ${ }^{18}$ Even though literature on fatigue in diabetics is rare, it does not mean that it is not present. The respondents in the current study narrated that they felt weak most of the times which made it difficult for them to conduct their daily chores or operate at optimum efficiency level at their workplaces.

Past research has shown that diabetic eye disease is the leading cause of new cases of legal blindness in American adults under the age of 65 years. ${ }^{19}$ In the current study, one female participant was completely blind and a number of them reported having poor sight that may progressively become worse with time. Persons with diabetes are at risk for visual loss due to diabetic retinopathy, glaucoma and cataracts. ${ }^{20}$ An approach to prevent visual loss which requires that patients with diabetes be systematically screened, referred and treated has been in use around the world. ${ }^{19}$ This approach is still in use even in Zambia because some respondents in the current research narrated how they attend the eye clinic for checkups. Despite the screening, a number of people still have sight problems and eventually became blind especially due to diabetes mellitus. There is a need for comprehensive eye care services that can address cataract, posterior segment disease and diabetes mellitus in the Zambian population. ${ }^{18}$

About $50 \%$ of men with T2DM are reported to have erectile dysfunction. Chronic renal failure has frequently been associated with diminished erectile function, impaired libido and infertility. ${ }^{21}$ In the current study, some of the male respondents experienced some sexual dysfunction of some kind. Libido effect was commonly reported. Improved techniques in diagnoses and treatment of impotence has given males with diabetes some hope in the management of this complication. ${ }^{22}$ Female sexual dysfunction or disorder is more difficult to define and specific studies in diabetes mellitus are limited. ${ }^{23} \mathrm{In}$ the current study, some of the women complained of vaginal itchiness and discharge.

The results of the current study show that physical sickness and hyperglycemia were one of the greatest experiences of adults living with T2DM. The physical sicknesses in the study can be controlled by good adherence to treatment guidelines. This good adherence to diabetic treatment guidelines prevents hyperglycemia. Physical sicknesses and hyperglycemia may be due to poor selfcare of the respondents and if not checked makes living with T2DM difficult. This study can be compared with the study in Thailand by Poosakew that aimed to determine the potential risk factors for hospitalization due to hypoglycemia among patients with diabetes. ${ }^{24}$ Poosakew's conclusion can be related to the findings in this study by suggesting that the physical sicknesses presented may be due to poor self-care which can be intensified by poor knowledge of the respondents on their condition. ${ }^{24}$ Physical sicknesses and hyperglycemia are predictors of diabetic hospital visits and admissions ${ }^{24}$ which some of our participants also narrated experiencing from time to time which makes these results consistent with the literature.

This study showed that patients with T2DM who had support from family and friends were healthier and happier than those that did not have the support. Other studies also show that social support from family provides patients with practical help and can buffer the stresses of living with the illness. ${ }^{25}$ Mayberry and Osborn ${ }^{26}$ in a 2012 study concluded that the participant reports of family members' non-supportive behaviors were associated with being less adherent to one's diabetes medication regimen. The respondents in the current study experienced a life of anguish as they were emotionally challenged by their loved ones who did not care for them.

Positive psychological health may sustain long-term coping efforts and protect patients with diabetes from the negative consequences of prolonged emotional disorders, illness perception and thus facilitating diabetes self-management behaviors and better physical health. ${ }^{27}$ Another study by Parildar and colleagues in 2015 which evaluated the relationship between depression, coping strategies, glycemic control and patient compliance in patients with T2DM demonstrated that patients with T2DM do experience depression. It was concluded that the likelihood of depression was frequent, and was associated with gender, educational status, coping strategies, duration of diabetes and patient compliance with treatment guidelines. ${ }^{28}$ Results from the current study share similar conclusions about some people living with diabetes experiencing depression because of their condition.

Diabetes and its complications bring about substantial economic loss to people with diabetes and their families and to health systems and national economies through direct medical costs and loss of work and wages. ${ }^{1}$ If left unaddressed, the growing prevalence of diabetes in the world will pose a tremendous challenge to the healthcare systems. ${ }^{29}$ In another study to quantify the increased health resource use and lost productivity associated with diabetes in 2012 in the USA, a prevalence-based approach indicated that the total estimated cost of diagnosed diabetes was $\$ 245$ billion, including $\$ 176$ billion in direct medical costs and $\$ 69$ billion in reduced productivity. ${ }^{30}$ The results in the current study show how the respondents admitted to having personal financial challenges because of diabetic treatment regimen. Challenges faced included costs to supplement the hospital monthly medication supply, costs to purchase the food needed to meet the diabetic nutrition therapy and costs for the purchase of glucose monitoring machines although not all the respondents encountered these challenges.

This study had limitations worthy of noting; first, the researcher's presence may have caused a response bias because male respondents could have withheld information to avoid embarrassment. Another bias could have been introduced by conducting the interviews at the diabetic clinic; there could have been respondents with more information elsewhere. Lastly, qualitative 
research data findings cannot be generalized to a larger population.

The results of this study highlight the experiences and challenges of some adults living with T2DM. These experiences and challenges especially of the psychosocial nature appear to be very overwhelming for the patients. Psychosocial therapy would make a great difference in the lives of people living with diabetes mellitus. There is a need to design an effective information education and communication program toward T2DM to educate people living with diabetes mellitus and the general population in Zambia. A suggestion to fund diabetic program and provide incentives for the diagnosed population such as medicine and blood glucose monitoring machines was also brought forward.

The experiences and challenges, especially of the psychosocial nature of some people living with T2DM appear to be very overwhelming for the patients. Their lives are clearly not easy making the need for both emotional and financial support evident from people around them. However, particular attention should be paid to the patient's self-care and psychosocial therapy. Self-care protocols should be tailored to complement the different patient types.

Contributors KFM developed the research topic and design. KFM also carried out proposal developmet, data collection and part of data analysis. PAB and $\mathrm{CJ}$ assisted in data analysis and supervision of the study from topic formulation to proposal development, data analysis, results and discussion presentations and finally, the conclusion. KFM wrote the manuscript with input from all authors.

Funding The authors have not declared a specific grant for this research from any funding agency in the public, commercial or not-for-profit sectors.

Disclaimer The views, thoughts, and opinions expressed in the text belong solely to the author/s, and not to the author's employer, organization, committee or other group or individual.

Competing interests None declared.

Patient consent for publication Not required.

Ethics approval This study was reviewed and approved by the University of Zambia Biomedical Research Ethics Committee (UNZA-BREC) Ref No: 013-06-16 on 17 August 2016 in Zambia.

Provenance and peer review Not commissioned; externally peer reviewed.

Data availability statement Data are available upon reasonable request.

Open access This is an open access article distributed in accordance with the Creative Commons Attribution Non Commercial (CC BY-NC 4.0) license, which permits others to distribute, remix, adapt, build upon this work non-commercially, and license their derivative works on different terms, provided the original work is properly cited, appropriate credit is given, any changes made indicated, and the use is non-commercial. See: http://creativecommons.org/licenses/by-nc/4.0/.

ORCID iD

Kunda Faith Mwila http://orcid.org/0000-0002-7209-9068

\section{REFERENCES}

1 World Health Organization (WHO). Global report on diabetes, 2016.

2 Olokoba AB, Obateru OA, Olokoba LB. Type 2 diabetes mellitus: a review of current trends. Oman Med J 2012;27:269-73.

3 Tuei VC, Maiyoh GK, Ha C-E. Type 2 diabetes mellitus and obesity in sub-Saharan Africa. Diabetes Metab Res Rev 2010;26:433-45.
4 Ministry of Health (MOH) 2008. 2008 STEPS report Zambia; prevalence rates of the common non-communicable diseases and their risk factors in Lusaka district. Zambia, 2008.

5 Nsakashalo-Senkwe M, Siziya S, Goma FM, et al. Combined prevalence of impaired glucose level or diabetes and its correlates in Lusaka urban district, Zambia: a population based survey. Int Arch Med 2011:4:1.

6 Azevedo M, Alla S. Diabetes in sub-Saharan Africa: Kenya, Mali, Mozambique, Nigeria, South Africa and Zambia. Int J Diabetes Dev Ctries 2008;28:101.

7 Hapunda G, Abubakar A, Pouwer F, et al. Diabetes mellitus and comorbid depression in Zambia. Diabet Med 2015;32:814-8.

8 Hall V, Thomsen RW, Henriksen O, et al. Diabetes in sub Saharan Africa 1999-2011: epidemiology and public health implications. A systematic review. BMC Public Health 2011;11:1.

9 Hapunda G, Abubakar A, van de Vijver F, et al. Living with type 1 diabetes is challenging for Zambian adolescents: qualitative data on stress, coping with stress and quality of care and life. BMC Endocr Disord 2015;15:1.

10 USAID. Qualitative Research Methods: A Data Collector's Field Guide Family Health International, 2005.

11 Agee J. Developing qualitative research questions: a reflective process. International Journal of Qualitative Studies in Education 2009;22:431-47.

12 Mowska JC. Qualitative mind: how to write a topic guide, professional training, FMRS, Genesis consulting, 2015.

13 Mack N, Woodsong C, Macqueen KM, et al. Qualitative research methods: a data collectors field guide, family health international, 2005.

14 Friese S. ATLAS.ti scientific software development $\mathrm{GmbH}$, Berlin. all rights reserved. manual version: 231.20151022. updated for program version: 7.5, 2015

15 Woods M, Paulus T, Atkins DP, et al. Advancing qualitative research using qualitative data analysis software (QDAS)? reviewing potential versus practice in published studies using ATLAS.ti and NVivo, 1994-2013. Soc Sci Comput Rev 2016;34:597-617.

16 Hycner RH. Some guidelines for the phenomenological analysis of interview data. Hum Stud 1985;8:279-303.

17 Measor L. Interviewing: a Strategy in Qualitative Research. In: Burgess R, ed. Strategies of educational research: qualitative methods. Lewes: Falmer Press, 1985.

18 Lindfield R, Griffiths U, Bozzani F, et al. A rapid assessment of avoidable blindness in southern Zambia. PLoS One 2012;7:e38483.

19 pp.Herman WH, Teutsch SM, Sepe SJ, et al. An approach to the prevention of blindness in diabetes. Diabetes Care 1983;6:608-13.

20 Saaddine JB, Honeycutt AA, Narayan KMV, et al. Projection of diabetic retinopathy and other major eye diseases among people with diabetes mellitus: United States, 2005-2050. Arch Ophthalmol 2008;126:1740-7.

21 Shindel A, Brant WO, Bochinski D, et al. 2014:Medical and surgical therapy of erectile dysfunction. In: Endotext [Internet]. South Dartmouth, MA: MDText.com, Inc, 2000.

22 Chu NV, Edelman SV. Erectile dysfunction and diabetes. Curr Diab Rep 2002;2:60-6.

23 Jackson G. Sexual dysfunction and diabetes. Int J Clin Pract 2004:58:358-62.

24 Poosakaew T, Kesssomboon P, Smith JF. Risk factors for hospitalization due to hypoglycemia in diabetic patients in northeast Thailand. J Diabetes Mellitus 2014;04:165-71.

25 Miller TA, Dimatteo MR. Importance of family/social support and impact on adherence to diabetic therapy. Diabetes Metab Syndr Obes 2013;6:421-6.

26 Mayberry LS, Osborn CY. Family support, medication adherence, and glycemic control among adults with type 2 diabetes. Diabetes Care 2012;35:1239-45.

27 Chew B-H, Shariff-Ghazali S, Fernandez A. Psychological aspects of diabetes care: effecting behavioral change in patients. World $J$ Diabetes 2014;5:796-808.

28 Parildar H, Cigerli O, Demirag NG, et al. Depression, coping strategies, glycemic control and patient compliance in type 2 diabetic patients in an endocrine outpatient clinic. Pak J Med Sci 2015;31:19-24.

29 Soewondo P, Ferrario A, Tahapary D. Challenges in diabetes management in Indonesia: a literature review. Global Health 2013;9:63.

30 American Diabetes Association. Economic costs of diabetes in the U.S. in 2012. Diabetes Care 2013;36:1033-46. 